\title{
EDUCACIÓN EN AMÉRICA LATINA: RETOS Y OPORTUNIDADES PARA LA FILOSOFÍA DE LA REGIÓN
}

\author{
EDGAR ESLAVA* \\ doi:10.11144/Javeriana.uph32-65.efro
}

\section{RESUMEN}

Enmarcadas dentro de las políticas de desarrollo para la región, las agendas educativas para América Latina representan compromisos que trascienden la mejoría en los resultados de pruebas específicas y que permean tanto las prácticas pedagógicas como los marcos conceptuales en los que estas cobran significado. El presente texto recoge algunos de los compromisos establecidos por dichas agendas y desarrolla las líneas generales que esperan y requieren la participación de la comunidad filosófica como garante y dinamizadora de la construcción de espacios de reflexión y acción sobre la educación.

Palabras clave: América Latina; educación; desarrollo; identidad; filosofía

* Universidad del Sinú, Montería, Colombia.

Correo electrónico: e_eslava@hotmail.com

Para citar este artículo: EslaVa, E. (2015). Educación en América Latina: retos y oportunidades para la filosofía de la región. Universitas Philosophica, 32(65), pp. 223-244. ISSN 0120-5323, ISSN en línea: 2346-2426, doi:10.11144/ Javeriana.uph32-65.efro 


\title{
EDUCATION IN LATIN AMERICA: CHALLENGES AND OPPORTUNITIES FOR PHILOSOPHY IN THE REGION
}

\author{
EDGAR ESLAVA
}

\begin{abstract}
Framed within the development policies for the region, educational agendas for Latin America represent specific commitments that transcend the improvement in the results of specific tests permeating both pedagogical practices and conceptual frameworks in which these ones acquire meaning. This paper covers some of the commitments made by these agendas and develops the general framework in which participation of philosophical community is expected and required as a guarantor and promoter of the construction of spaces for reflection and action on educational system. Keywords: Latin America; education; development; identity; philosophy
\end{abstract}


América latina cuenta actualmente con un buen número de agendas multilaterales propuestas para mejorar la calidad, cobertura y competitividad de sus sistemas educativos, con un énfasis especial en la formación en ciencia y tecnología. Ejemplo de ello son la Declaración de São Pablo (2002), la Declaración de Buenos Aires (2007) y la Declaración de Lima (2014), en las que los gobiernos de los países de la región se comprometen con la promoción de sistemas educativos que garanticen una educación de calidad con cobertura universal, sin discriminaciones, cuyas necesidades económicas se vean cubiertas en parte por la asignación de ingresos significativos en términos del PIB de cada país; que aseguren la incorporación de nuevas tecnologías, el desarrollo sostenible y la ciudadanía global; que promuevan la integración regional y den un lugar y un tratamiento digno a los profesores, a quienes se reconoce como los primeros agentes de promoción y concreción de los compromisos de una educación entendida desde la doble perspectiva de bien público y de derecho fundamental.

Es evidente que gran parte de las agendas educativas debe ser cubierta por los gobiernos, quienes han de garantizar no solo los recursos y la puesta en marcha de políticas que avancen hacia el cumplimiento de los acuerdos, sino la ejecución de planes concretos que garanticen la difusión a la población; el seguimiento sistemático y permanente, tanto por parte de las agencias estatales correspondientes como por parte de la sociedad civil y, por encima de todo, la repercusión en una educación de calidad. Esta ha de corresponder con las necesidades particulares de cada país y ha de permitir a sus ciudadanos fortalecer competencias tanto laborales como de crecimiento personal, a fin de alcanzar una vida digna.

Sin embargo, no es lógico pensar que todo el peso de los compromisos recae únicamente en los gobiernos. Trabajar por la construcción y consolidación de planes, políticas y estrategias que permitan la concreción de los compromisos establecidos en las agendas corresponde y compromete a los entes no gubernamentales de revisión y fiscalización, a las universidades y centros de formación del profesorado, a las escuelas y las familias, a los estudiantes. En pocas palabras, corresponde a la sociedad en pleno de quien la educación es un derecho y un bien.

Es en la sociedad donde los deseos se tornan en objetivos, y las políticas, en planes. Y dado que esta requiere de la ayuda y de la participación de todos sus 
estamentos, es allí, precisamente, en donde la filosofía, los filósofos, deben encontrar su lugar, participando en la construcción de los marcos de análisis e interpretación de nuestras realidades latinoamericanas, que son varias y, con todo, una. Nos compete la tarea de comprender nuestra realidad, destacar y defender su diversidad y participar en la construcción de un marco interpretativo que nos permita un diálogo inteligente y efectivo, que permee las dimensiones políticas, técnicas y pedagógicas y que permita el paso de nuestras sociedades actuales, desinformadas y desinteresadas, a una sociedad responsable consigo misma, por medio del desarrollo de sus sistemas de educación, de sus necesidades, de sus alcances y de su evaluación.

Esta necesidad de participar en el desarrollo de modelos educativos consistentes y efectivos es una excusa perfecta para dejar de lado esa abulia que ha caracterizado la relación filosofía-educación desde finales de los años 70 del siglo pasado, cuando parece que los filósofos latinoamericanos perdimos la fe en la posibilidad de impactar las políticas y prácticas educativas y, con ella, la fe en nuestra tarea.

El presente documento ofrece una lectura, con una perspectiva regional, tanto de los compromisos como de las alternativas de solución, acerca de la relación entre filosofía y educación dentro del marco de los programas y propuestas de las agencias internacionales y de las políticas que de ellas se derivan para los gobiernos participantes. Se ha dejado para el cierre algunas ideas puntuales acerca de cómo dinamizar esta relación y, más importante incluso, de cómo dar respuesta desde la filosofía, desde las filosofías, a una discusión que ha de permanecer abierta a la espera de nuevos desarrollos tanto en las preguntas que se abren como en los marcos de interpretación desde los que se plantean sus posibles respuestas.

\section{Educación: los compromisos globales}

INICIEMOS NUESTRO RECORRIDO con una mirada a la piedra angular de toda agenda actual de cooperación internacional, los denominados "objetivos del milenio" de la UNESCO. Dichos objetivos, generales por definición, fueron introducidos como punto de partida para el desarrollo e implementación de programas y acciones nacionales e internacionales y se han transformado en los referentes de evaluación del progreso hacia la erradicación de las manifestaciones de la pobreza y del grado de aproximación al bienestar general de los habitantes del planeta. 
Los objetivos del milenio son:

1. Erradicar la pobreza extrema y el hambre.

2. Lograr la educación primaria universal.

3. Promover la igualdad de géneros y empoderar a la mujer.

4. Reducir la mortalidad infantil.

5. Mejorar la salud materna.

6. Combatir el VIH/SIDA, la malaria y otras enfermedades.

7. Asegurar sostenibilidad ambiental.

8. Desarrollar una asociación global para el desarrollo.

Si bien a primera vista pudiese parecer que la educación solo se hace presente de manera directa en el segundo de los objetivos, una mirada más detallada demuestra que esta se encuentra en la base de la solución de los otros siete objetivos. La erradicación de la pobreza no es solo un asunto de abastecimiento de alimentos y de políticas de desarrollo sostenible, sino de educar en el uso de los recursos disponibles, de acercar a las comunidades al conocimiento que les permita intervenir de forma directa en sus entornos, a fin de alcanzar mejores condiciones de vida. El empoderamiento de la mujer y la reducción de mortalidad infantil tienen injerencia tanto política como educativa, pues a la par de crear legislaciones justas y no discriminatorias, se debe trabajar en programas que, de un lado, eduquen a las familias en temas de sanidad y prevención y, de otro, enseñen que la igualdad de género no se limita tan solo a que las mujeres puedan acceder a los centros de atención de salud y alimentación, sino que se les permita la oportunidad de completar su educación, optar por los trabajos que deseen desempeñar y que estos sean reconocidos social, política y económicamente, con las mismas condiciones que el trabajo de los hombres. También será la combinación de legislaciones y sistemas educativos pertinentes la que permita trabajar en pos de los objetivos de combatir enfermedades y asegurar sostenibilidad ambiental, en este caso haciendo énfasis en las dimensiones científico-técnicas de los programas políticos y educativos.

Solo mediante la inclusión de la educación como parte de la agenda de solución a estos problemas, esta podrá contribuir al desarrollo pleno de la dignidad humana y ser consecuente con su función y su propósito. Esta educación debe superar las perspectivas que caracterizaron a los modelos educativos durante los 
dos últimos siglos, centradas en la transmisión y acumulación acrítica de contenidos; además, debe trabajar bajo un modelo que potencie la identificación y resolución de problemas y la responsabilidad frente al individuo, al ambiente y a la comunidad.

La educación tiene calidad si los estudiantes tienen la oportunidad de conocer y vivir [sus] derechos -lo que significa el aprendizaje no solo de conocimientos y habilidades, sino por encima de todo el desarrollo de valores, actitudes y comportamientos. Aunque es necesario, no es suficiente que los estudiantes desarrollen sus capacidades cognitivas y aprendan habilidades básicas para conseguir un trabajo adecuado y gratificante. También es necesario que desarrollen habilidades sociales para ser capaces de vivir armoniosamente en un mundo plural, que alcancen un desarrollo afectivo balanceado y una conciencia moral que les permita actuar con autonomía y responsabilidad. (UNESCO, 2007)

Una educación de calidad, entonces, solo se alcanzará si el proceso educativo se centra en el desarrollo de competencias (o habilidades, o capacidades, como quiera denominárseles) que permitan un aprendizaje que perdure a lo largo de la vida en las dimensiones del conocer, el hacer, el crecimiento personal y la convivencia.

\section{Con América Latina en la mira}

Nuestro continente no escapa, como es natural, a la necesidad de crear mecanismos para alcanzar todos los objetivos del milenio. Tampoco puede escapar a los compromisos que ello implica. Nuestra realidad nos hace particularmente sensibles a algunas necesidades y nos obliga a repensar las acciones que han sido implementadas para satisfacerlas, ya que, más allá de las diferencias, todo lo que hacemos para superar nuestras dificultades tiene repercusiones regionales que afectan a nuestras comunidades locales y a nuestros vecinos y hermanos, aunque esa noción de hermandad resulte ser problemática para defender e incluso definir. El estudio de América Latina es el análisis de patrones culturales y necesidades sociales similares, así como de aproximaciones muy semejantes a la solución de los problemas. Esto es, en parte, resultado de herencias históricas comunes, del uso compartido de referentes y modelos de acción y de nuestra compleja noción de la identidad nacional y regional. En palabras de Carlos Fuentes (2012): 
Le dimos la espalda a la cultura española porque era la cultura de la Colonia; le dimos la espalda a la cultura indígena, porque la considerábamos salvaje o bárbara, de tal manera que nos quedamos un poco huérfanos, a la intemperie, por un acto propio nuestro. Nos dedicamos a imitar a Europa y los EE.UU. -que es lo que yo llamo las repúblicas Nescafé, de democracia instantánea que creamos en el siglo XIX-. Fue indispensable un acto de reflexión para llegar a conseguir la identidad propia, para recoger los sinos de la tradición y darnos cuenta de que somos europeos, mediterráneos, judíos, griegos, romanos, indígenas, negros y que somos sobre todo mestizos. Pero ahora tenemos que pasar de la identidad a la diversidad. A pensar, política y socialmente, la diversidad. A respetar la diversidad sexual, política, moral, religiosa de los ciudadanos. ${ }^{1}$

Con la mira puesta en la educación, es evidente que compartimos necesidades. Si bien la mayoría de los países latinoamericanos ha alcanzado niveles de cobertura de la educación primaria cercanos al 95\%, las de educación media y superior está aún lejos de esos porcentajes (UNESCO, 2011). Con apenas un par de excepciones, las tasas de deserción escolar siguen siendo muy altas en nuestros países y el porcentaje de estudiantes que culminan la educación media e ingresan a la superior apenas supera el 30\%, con pocas alternativas que garanticen algún tipo de formación postsecundaria. A pesar de algunos esfuerzos destacados, América Latina sigue teniendo altos índices de disparidad de género frente al acceso al sistema educativo, pues aunque es mucho mayor el porcentaje de varones que tiene la oportunidad de una educación básica y media completas, son las mujeres quienes encabezan las estadísticas de matrícula y graduación de la educación superior (UNESCO, 2008a; PNUD, 2010). Otro tipo de disparidad está asociado con la marcada tendencia a mantener los centros de estudio en las zonas urbanas, lo que se convierte en un enorme obstáculo para alcanzar los objetivos de cobertura y calidad en una región con una población principalmente rural. Frente a los planes de estudio, casi la totalidad de los gobiernos latinoamericanos ha optado por currículos centrados en una instrucción que privilegia los contenidos de las asignaturas, mientras que los indicadores internacionales de calidad dirigen la atención a la medición de habilidades transdisciplinarias; esto provoca

1 Entrevista concedida al diario El Comercio y publicada en Internet el 15.05.12. 
que nuestros resultados en las pruebas internacionales sean pobres, que seamos poco competitivos y que se reste a nuestros jóvenes oportunidades de acceso a programas de becas y subvenciones de estudios. Finalmente, aunque los números muestran una tendencia a la mejoría, la inversión en educación parece no ser una prioridad de todos los gobiernos, en particular en cuanto a la formación y condiciones del personal docente, lo que tiene implicaciones directas tanto en la calidad de los programas académicos como en las condiciones de vida de los maestros y directivos (UNESCO, 2014a, 2014b).

Por el lado de las soluciones, se han dado algunas respuestas interesantes y efectivas a los problemas que compartimos, pero no logramos estar todos a la par, con marcadas diferencias que reflejan las realidades particulares de cada uno de los países: sus niveles de pobreza y marginalidad, sus situaciones políticas y de seguridad interna, sus posibilidades y pretensiones de exposición ante la comunidad internacional y otras variables que hacen difícil la comparación de resultados y la construcción de planes de acción conjuntos que permitan un crecimiento regional estable y homogéneo. Pero más allá de las diferencias y los desarrollos idiosincrásicos resulta indispensable reconocer que se debe trabajar individual y conjuntamente para garantizar una educación de calidad inclusiva desde la perspectiva de los derechos humanos, es decir, se debe permitir no solo posibilidades de acceso similares a los centros educativos de las comunidades más vulnerables o discriminadas, sino la garantía de igualdad de todos los ciudadanos. Se debe también reconocer el papel de los profesores como mediadores y garantes del derecho a la educación, y como tales han de asegurárseles unas condiciones de vida y de ejercicio profesional acordes con las expectativas de los resultados de su labor.

Con todas estas necesidades en mente, los estudios regionales de medición de progreso hacia los objetivos de una educación de calidad, entendida en los términos expresados anteriormente, han sugerido ciertas metas específicas para los países de la región. Para superar nuestros vacíos más notables, para dar alcance a los compromisos y agendas que declaran el interés por hacernos competitivos en el panorama internacional y para que toda la población, en especial la menos protegida, alcance los niveles básicos de educación que le permitan "conocer y vivir sus derechos", se han de desarrollar competencias que permitan aprendizajes sostenidos en el tiempo, pues solo así podremos afirmar que en el continente sus habitantes gozan de una plena ciudadanía. 
Para trabajar en esta dirección es necesario plantear tareas pertinentes y concretas que apunten a la solución de la problemática característica de nuestros países. En ese sentido, la Organización de Estados Iberoamericanos ha presentado un interesante estudio que se constituye como una herramienta de apoyo para definir la visión de la educación en la región en la segunda década del presente siglo (OEI, 2008). De acuerdo con dicho documento, las metas generales de la educación en la región son:

1. Comprometer a la sociedad con la educación.

2. Educar en la diversidad.

3. Extender la educación temprana.

4. Universalizar la educación básica y mejorar su calidad.

5. Asegurar que todos los alumnos alcancen las competencias básicas.

6. Incrementar el acceso de los jóvenes a la enseñanza postobligatoria.

7. Conectar educación y empleo a través de la Educación Técnico Profesional.

8. Educar a lo largo de toda la vida.

9. Cuidar el desarrollo profesional de los docentes.

10. Contribuir a la configuración del espacio iberoamericano del conocimiento y la investigación científica.

11. Conseguir más recursos para la educación e invertir mejor.

Muchas de estas metas son derivadas de los propósitos globales que ya han sido revisados y reconfiguradas a la luz de los resultados de los estudios sobre el estado de nuestras prácticas, políticas e instituciones educativas. Estas metas pueden ser clasificadas de acuerdo con problemas los específicos a los que ellas refieren, lo que nos permitirá más adelante construir una propuesta de acciones concretas para los filósofos de la región.

De estas metas, unas abordan la educación como un bien público. A esta categoría pertenecen la necesidad de comprometer a la sociedad con la educación, la extensión de la educación temprana y la universalización de la educación primaria, el incremento de acceso a la educación postsecundaria (cuya definición varía de país a país), la conexión entre empleo y educación, y la necesidad de incrementar e invertir adecuadamente los recursos financieros que llegan a los sistemas educativos. Estos objetivos constituyen parte de la agenda política hacia la con- 
secución de una educación de calidad y, por ello, se convierten en el derrotero de los gobiernos interesados en alcanzarla y en un compromiso del sector privado que ha de participar en la ampliación de la oferta educativa. Sin embargo, estos objetivos solo se alcanzarán si se crea un interés real por demostrar la importancia, validez y necesidad de invertir en educación, de generar demanda para el tipo de empleos que requiere preparación formal, ya sea académica o técnica. Es necesario que a las políticas de cobertura las acompañen políticas de desarrollo social, de las cuales todos los estamentos sociales deben hacerse partícipes, de modo que hagan visibles y ofrezcan alternativas de solución a las necesidades específicas en cuanto a infraestructura, acceso a los centros y recursos educativos y capacitación del personal docente en cada centro educativo, en cada población, en cada país.

Otro grupo de objetivos refiere a la calidad y contenido de la educación. A esta categoría pertenecen el aseguramiento de adquisición de competencias básicas, la educación en la diversidad, el consolidar un aprendizaje durante y para toda la vida y el garantizar la idoneidad de los agentes directos de intervención en el aula: los docentes. La consecución de estas metas implica un trabajo dirigido a la concreción de sistemas educativos coherentes con las realidades nacionales y que combinen ciertas características de homogeneidad y heterogeneidad. Los contenidos educativos han de ser similares para todos los ciudadanos sin distingos de raza, credo, ubicación geográfica o nivel económico; también, deben estar estructurados de forma tal que garanticen una base compartida de competencias mínimas, al final de cada ciclo educativo, deseables en todos los estudiantes. Estas competencias mínimas deben preparar a los ciudadanos para acceder y hacer uso adecuado del conocimiento y de las herramientas contemporáneas, para su desarrollo y transmisión, para posibilitar y alentar la resolución de conflictos, para la convivencia pacífica y para el crecimiento y el análisis crítico de los valores culturales y personales que permitan el fortalecimiento de la personalidad y la expresión de las cualidades e intereses personales, los cuales, llegado el momento, permitirán que la toma de decisiones por parte de un individuo se haga de forma tal que su resultado vaya dirigido a mejorar la calidad de vida personal y de la comunidad.

De otra parte, además, la oferta educativa ha de ser heterogénea, diferenciada. Debe partir del reconocimiento de la diversidad característica de nuestra demografía y de nuestra cultura. Si esta diversidad forma parte de los planes de estudio se evidenciará un conocimiento de las realidades locales, comprensión de 
sus características propias y de la forma en que participan del contexto regional y mundial al que no solo no podemos escapar, sino al que debemos reconocer y del que debemos participar. Nuestros sistemas educativos deben abrir las puertas a la interpretación libre, no anárquica ni desencajada, de sus contenidos y sus posibles formas de aplicación, que no repita acríticamente modelos foráneos y los imponga ciegamente en los contextos locales. Heterogéneos han de ser también los objetivos de cada etapa dentro del sistemas de educación, de modo que se privilegie la formación en competencias básicas en la escuela primaria, el desarrollo de la mentalidad crítica y el compromiso personal en la educación media y las competencias analíticas y laborales en la educación superior. Como resultado de ello, se tendrán niños y niñas inquietos frente al mundo y capacitados para comprenderlo, jóvenes que exploran la vida a partir de un claro conocimiento de quiénes son y lo que esperan de sus vidas y adultos generadores de respuestas, de empresas, de nuevos modelos de interacción e integración social y resolución de conflictos. Si se espera calidad en los contenidos de la educación y en la educación como sistema, estos deben definirse con seriedad y propiedad, contando con la participación de quienes van a determinar y ejecutar las políticas, pero también, indispensablemente, de quienes van a ser sus directos beneficiarios y partícipes.

La última categoría de objetivos dirige la atención a la configuración del "espacio iberoamericano del conocimiento y la investigación científica" y expresa metas que, a la vez que involucran la creación y sostenimiento en un tiempo de políticas claras de educación en ciencia y tecnología, nos impele a la construcción de la unidad regional.

Por el lado de la integración regional, puede afirmarse que la creación de un espacio latinoamericano de conocimiento es una extensión de las necesidades evidenciadas en la categoría de las metas de la educación como bien social, en donde lo local, de un lado, se circunscribe ahora a América Latina como región y no a cada una de las naciones que la componen; de otro, se apersona de las agendas de los gobiernos centrales por medio de las agencias de integración regional que trabajan por los intereses compartidos. Como consecuencia, el reconocimiento de particularidades sociales y culturales ha de trascender las fronteras nacionales y la puesta en marcha de proyectos comunes, y ha de descansar sobre la necesidad de una colaboración franca y abierta entre vecinos. 
Sin embargo, esta dimensión de la tarea no se agota en el trabajo en conjunto por la resolución de aquellos problemas propios reconocidos en los otros, sino que implica el reconocer a nuestra región como campo de conocimiento, por definición y estructura, complejo y rico en diversidad y, aparentemente, olvidado al escribir nuestra historia. El eje académico en América Latina es, en los pocos países en que existe como tal, apenas un brote, una situación que contrasta con las miradas que sobre nosotros mantienen diversos centros académicos europeos y norteamericanos. Tal parece que lo que otros encuentran en nosotros no nos es, por familiar o cercano, digno de atención. Recuperarnos como objetos de reflexión facilitará la tarea de ofrecer soluciones a nuestros problemas y hará que las respuestas ofrecidas estén tan cerca a nosotros como las preguntas que abordan.

Por el lado de la formación en ciencia y tecnología, que es específica en cuanto a contenidos y calidad de los programas y complementaria de las agendas de educación como bien público, el reto es múltiple. Por una parte, se espera alcanzar unos niveles de apropiación y desarrollo de las ciencias y las tecnologías que pongan a la región a la par de otras regiones del mundo. Este es, diríamos, el objetivo externo. De otra parte, se requiere hacer recurrente y sistemático el uso de los conceptos, prácticas y resultados de las ciencias para proponer soluciones a algunos de nuestros problemas más acuciantes: la violencia, la pobreza, la falta de desarrollo en las regiones rurales. En este sentido, es necesario trabajar por una ciudadanía científicamente informada, con capacidad para incorporar en la cotidianidad las tecnologías que tiene a su disposición y preparada en el uso, alcances y límites de las ciencias y las tecnologías como agentes de desarrollo social. Si bien es cierto que no podemos cometer el error de creer que las ciencias por sí solas resuelven problemas, o que los métodos e intereses científicos superan siempre y en cualquier situación a otros provenientes de diferentes direcciones, tampoco podemos, simplemente, negarnos a avanzar en el desarrollo de áreas del conocimiento que han mostrado su efectividad como herramientas de trabajo, como políticas de desarrollo y como instrumentos pedagógicos. El desarrollo sólido en ciencia y tecnología puede no ser la respuesta a todas nuestras necesidades, pero no formar ciudadanos competentes en ciencias y tecnologías es declarar la intención de seguir a la saga de otras naciones y otras regiones del planeta. 


\section{Un lugar para la filosofía}

VARIOS DE LOS COMPROMISOS Y METAS que de cara a la educación se presentan a los filósofos latinoamericanos han sido ya enunciados de forma general en los apartados precedentes. En esta sección final mostraremos el tipo de colaboración o los pasos firmes que podemos dar para cumplir los objetivos propuestos a favor de una educación de calidad en América Latina. Para ello haremos uso de las categorías anteriormente definidas, cada una de las cuales ofrece marcos particulares de acción y permite definir aplicaciones y espacios concretos de intervención para una filosofía comprendida como actividad multidimensional, que traspase los límites de la discusión académica y se convierta en acción comprometida y efectiva socialmente.

Frente a la educación como bien público, hemos enfatizado que el desarrollo social y la inclusión solo se pueden alcanzar si se mantienen abiertos el diálogo y el debate y, además, si se respetan puntos de vista alternativos. En este contexto, es fundamental que la filosofía se haga partícipe ayudando a configurar una visión de conjunto de las necesidades particulares de la región, a fin de que los intentos de solución ofrecidos no se dispersen y los esfuerzos no se malgasten. La labor de los filósofos es, entonces, la crítica. Se debe interrogar a las políticas, sus intereses, sus alcances, sus grupos objetivo y sus puntos de partida. Debemos ser observadores juiciosos, atentos; participar no solo con una mirada filosófica a la política sino a partir de una filosofía como política. Los debates desde la academia deben complementarse con la participación de los filósofos como grupo disciplinar en las mesas de trabajo sobre políticas educativas, de manera que presenten y representen no una voz sino la voz de muchos. El filósofo como político debe dar contenido a la noción de sociedad que subyace a los intentos por transformarla, a la noción de educación cuya calidad se desea mejorar y a la noción de ciudadano cuya construcción da sentido a la tarea de educar. Los filósofos están llamados a dar luces sobre los requisitos que impone una realidad global sobre nuestras acciones locales.

A manera de ejemplo, cabe mencionar el cambio en la orientación de mediciones del bienestar social a partir de una relectura filosófica de los modelos tradicionales. En su versión clásica, la medida del bienestar, tanto a nivel individual como nacional, estuvo asociada de manera directa al PIB. De acuerdo con esta lógica, un país se encuentra en un estado satisfactorio si su PIB se encuentra por encima 
de ciertos niveles determinados por las teorías econométricas al uso, y se entiende que el bienestar de una nación se distribuye estadísticamente entre sus pobladores, de modo que a países con un alto PIB corresponden directamente individuos con altos niveles de bienestar. Sin embargo, a partir de la propuesta adelantada por Sen (1980) y luego extendida por Nussbaum $(1993,1997)$ en términos de capacidades, no solo se puso en duda la posibilidad de que un indicador económico pudiese dar cuenta de una condición tan compleja como el bienestar, sino que se dio inicio a una nueva etapa en la construcción de indicadores que den cuenta de la multiplicidad de variables que el modelo convencional simplemente desconocía. Como resultado, incluso agencias centradas en el desarrollo económico ahora cuentan con indicadores de bienestar que ponen al mismo nivel de importancia las condiciones materiales de subsistencia (ingresos, trabajo, vivienda) y condiciones de calidad de vida más cercanas al desarrollo individual (educación y competencias, relaciones sociales, seguridad personal) (OECD, 2011). En este sentido,

[1]a aproximación desde las capacidades ofrece entonces un amplio marco normativo para conceptualizar y evaluar el bienestar individual y los acuerdos sociales en cualquier contexto o sociedad particular. No es una teoría de la justicia completa, pero trata con cuestiones acerca del balance entre libertades e igualdad que han caracterizado el trabajo en justicia social desde finales del siglo dieciocho. Sen (1980) formula la pregunta central "¿Igualdad de qué?" Como él mismo lo explica, todas las teorías igualitarias que han resistido al paso del tiempo ponen el asunto [en términos de] la igualdad de algo, por ejemplo, de ingresos, niveles de bienestar, derechos o libertades. En educación esta cuestión emerge en el trabajo filosófico y sociológico sobre cómo teorizar y analizar la provisión de oportunidades de aprendizaje equivalentes. (Walker \& Unterhalter, 2007, p. 3)

Reconocer y hacer uso de esta capacidad crítica de la filosofía es recuperar su esencia como forjadora de cultura y ponerla al servicio de nuestra cultura. Estamos en mora de reavivar esta llama. La idea de la filosofía como política exige revisar nuestra participación en la construcción de la unidad regional, y si bien la filosofía puede convertirse en una pieza clave para la discusión de nuestras necesidades, nuestras posibilidades, nuestros intereses, nuestros problemas y nuestros intentos de solución de los mismos, tal vez la tarea fundamental en esta 
dirección sea la de dar alcance al nosotros que subyace a estas variables. Superando una definición superficial en términos geográficos, se debe participar en la construcción de una definición más rica y elaborada, a partir de la cual cobre sentido una noción de ciudadanía y de participación ciudadana consistente con nuestras realidades, diversas y comunes. Esta tarea de la filosofía como espejo ha de ayudar a dar forma a una aproximación original y auténtica de nuestra identidad, de nuestro papel como región en la dinámica geopolítica global y de nuestro inescapable compromiso con la definición de nosotros mismos.

Este ideal no es nuevo, pero ha de ser restaurado permanentemente para que su fuerza no se apague, para que la tarea no caiga al olvido. Para ello contamos con voces venidas desde los extremos cartográficos del continente que intentan avivar el ideal de la búsqueda de nuestra identidad. Así, contamos con los siguientes aportes de Zea y de Dussel:

América, como todos los pueblos, irá tomando conciencia de su realidad, mediante un movimiento dialéctico en el que se enfrentan las opiniones de Europa sobre el ser y las que ella misma deduce al confrontarlas con lo que es en sí misma. Por un lado está lo que Europa quiere que sea y por el otro lo que en realidad es. Por un lado la serie de justificaciones que se da a sí mismo un europeo al imponer su dominio político, cultural y social sobre Améri$\mathrm{ca}, \mathrm{y}$ por el otro las reacciones del americano frente a estas justificaciones que le menoscaban. (Zea, 1954, p. 83)

Nuestra hipótesis es la siguiente: Aún para la comprensión radical de cada una de nuestras culturas nacionales se deberá contar con las estructuras de la cultura latinoamericana, como su horizonte. No puede postergarse el análisis de Latinoamérica para un futuro remoto, cuando el estudio de nuestras culturas racionales haya terminado. Es un absurdo en morfología cultural, ya que son las estructuras del todo las que explican la morfología de las partes. En fisiología se estudia la totalidad funcional del cuerpo, lo que permite descubrir los órganos y sus actividades complementarias. (Dussel, 2006, pp. 111-112)

De Zea a Dussel, la construcción dialéctica de la identidad latinoamericana es un debate entre internalismo y externalismo que se espera que resulte en una definición clara y sin ambivalencias de lo que nos es propio, digamos, de nuestro ser común. Si bien la tarea no ha sido concluida, hoy se ve aumentada con nuevos 
retos, pues, contrario a los que sucede en otros continentes, nuestras guerras entre naciones vecinas han venido cesando y nuestras excusas para la violencia no están centradas en diferencias de fe o de color, sin que ello implique que estas hayan dejado de existir. De otra parte, las brechas de desigualdad que nos caracterizan no tienen paralelo y en todos nuestros países el pobre muere desconocido, víctima de la apatía y del desinterés de sus conciudadanos, que a pesar de no parecerlo, lo son. Y si bien por todos los medios nos llega el mensaje de que en la educación está la solución, seguimos a la espera de formas concretas y efectivas de hacer de esta promesa una realidad.

Frente a la calidad y el contenido de la educación, la filosofía como espejo ha de contribuir con la articulación de respuestas a las preguntas sobre quiénes somos y quiénes queremos ser. Además, ha de aportar contenido a la idea de identidad cultural regional a partir de la doble direccionalidad implicada en la su construcción a partir de las identidades nacionales y de estas como resultados locales de una historia cultura y desarrollo compartidos. Así mismo, se espera una contribución filosófica al significado de lo que es aprender a vivir juntos, habida cuenta de que nuestras actuales agendas tienden a ocultar, o al menos a no hacer explícitos, los lenguajes, tradiciones, contextos y esperanzas de los pueblos latinoamericanos, tanto al interior de cada nación como en su conjunto. En este sentido, el llamado que se le hace a la filosofía en general bien pudiese haberse hecho como clamor a los filósofos de la región.

La verdadera paz no es simplemente el resultado de negociaciones políticas o de acuerdos estratégicos entre personas y estados, sino el trabajo de seres humanos con el entrenamiento y la pasión para buscar la verdad y hacer el bien. La paz verdadera entonces tiene una base antropológica, que es el der humano humanizado comprometido con la verdad y en bien como propiedad común de toda la humanidad. Esta es precisamente la importancia fundamental que la filosofía ha de tener en la promoción de la paz en el mundo de hoy, dado que representa la forma de conocimiento que de manera más diáfana enseña a los seres humanos que el deseo de lo bueno y lo verdadero va de la mano con el deseo de coexistir en paz con uno mismo, con la naturaleza y con los demás. (UNESCO, 2014c, p. 6)

Así, debemos garantizar que, con los ojos en lo universal de la filosofía, atendemos a la pregunta por la particularidad de nuestros problemas, cuya respuesta 
irá seguramente asociada a la comprensión de nuestra historia compartida y de nuestro presente, lleno de condiciones que, a la vez, nos aparta y nos acerca, en ocasiones de forma explícita, pero en muchas otras de manera menos directa, pero no por ello menos relevante. Una educación en y para la unidad regional no puede ser simplemente la suma de sistemas educativos que comparten una lengua y ciertas características de contexto, sino una que promueva la consolidación de valores compartidos entre hermanos a los que, como tales, el habitar en vecindad geográfica no evita que hayamos crecido distantes unos de otros.

Conscientes de nuestra tarea de educar en la diversidad y de alcanzar un aprendizaje que dure toda la vida, debemos ofrecer alternativas que, dentro del marco general de una educación de calidad, sean válidas y aplicables en nuestro contexto. A partir de esta necesidad de generar identidad, la filosofía tiene mucho que aportar al contenido y a la calidad de la educación en la región, una tarea que ha de asumirse no solo desde la educación en la filosofía, necesaria como es, o desde la mirada de una filosofía de la educación, sino a partir de la filosofía como educación, la cual hace uso de las herramientas propias de la disciplina y las pone al servicio de quienes definen las políticas y agendas educativas, de quienes las implementan, de quienes las evalúan y de aquellos para quienes estas son creadas en primer lugar. Por supuesto, no van a ser los filósofos quienes definan los contenidos específicos de los currículos disciplinares en los planes de estudio de los centros educativos de toda la región, pero así como nos debemos comprometer con la mirada y análisis críticos de las políticas en educación y con la definición de la identidad regional, también debemos, como disciplina, participar del debate sobre las competencias que deben formar parte de la educación regional, de los contenidos que las soportan, de los proyectos transversales que les dan forma y del ciudadano que queremos, que necesitamos, como resultado de la educación que ofrecemos.

Mucho se habla de aprender de las experiencias de aquellos países que en poco tiempo han escalado posiciones en las mediciones internacionales de calidad educativa. Finlandia, Singapur y Corea son algunos de los nombres que se mencionan frecuentemente como ejemplos a seguir, pero una visión acrítica de los contextos en que estos modelos educativos son diseñados e implementados puede agregar a la ansiedad generada por estar donde se quiere, el ver cómo la estrategia escogida falla una y otra vez. Una filosofía crítica que analiza contextos, que participa de las discusiones, que ofrece alternativas para acercar los modelos a 
las necesidades reales y que convoca a la unidad alrededor de sus propuestas, es una filosofía que, en tanto educación, debe y puede ser promovida a lo largo de las etapas de formación académica de los ciudadanos, dentro y fuera del ámbito escolar. Y si hay una lección que deba ser aprendida de los modelos educativos más efectivos es que a la base de cada uno de ellos hay un claro horizonte de ciudadanía; un acuerdo explícito sobre el tipo de ser humano, al nivel más global, y de ciudadano, al nivel local, al que dicho modelo responde y sobre el que son juzgadas sus estrategias, sus metodologías, los lugares que asigna a cada uno de los estamentos que intervienen en su desarrollo, y las herramientas que ofrece a cada de ellos para cumplir con la tarea que le es encomendada. He aquí un claro espacio para que la filosofía ofrezca su servicio a la construcción del ideario, de la ruta y de la lógica de evaluación del avance de los proyectos educativos de la región.

Finalmente, frente a la formación científica y tecnológica, la consecución de una educación de calidad debe abordarse desde la perspectiva de la relación de los habitantes de la región con las ciencias y las tecnologías. ¿Es necesario definir categorías especiales que permitan dar cuenta de nuestra relación con las ciencias, o nos basta con un discurso general y tradicional? Sabiendo que frente a las tecnologías somos básicamente consumidores y que queremos avanzar hasta convertirnos en productores originales, ¿será suficiente reconstruir la historia de quienes van al límite del conocimiento? ¿Debemos transformar y reinventar la historia de las naciones que han superado brechas tecnológicas y hoy se encuentran a la vanguardia? ¿Existen caminos intermedios que no hemos explorado? $\mathrm{Si}$ los desarrollos conceptuales nos son ajenos, tal vez nos falte aún algo de ingenio, de interés, de creatividad para transferir y adecuar el conocimiento a la luz de objetivos y resultados relevantes. Si seguimos aplicando ciencias y tecnologías que no son pertinentes porque no surgen de las necesidades de nuestro contexto, quizá podamos hacerlas nuestras adaptándolas y convirtiéndolas en soluciones a nuestros problemas. Si la educación, en general, y la educación en ciencia y tecnología, en particular, llega a pocos y se convierte en parte de la estructura conceptual y de resolución de problemas de muchos menos, es claro que estamos en mora de ofrecer alternativas para que su apropiación social sea consistente con el impacto que se espera de su implementación.

En otro lugar he desarrollado una aproximación inicial a algunas alternativas que desde la filosofía surgen para la construcción y revisión de propuestas de 
formación en ciencias naturales ${ }^{2}$. En el contexto de la presente discusión, baste decir que, así como resulta ideal que al interior de las instituciones educativas se establezcan con claridad los objetivos de la formación científica en cada una de las etapas de escolaridad -en la escuela primaria contacto con el mundo natural e interés por explorar, en la escuela secundaria formación en competencias formales, sociales y lingüísticas que permitan dar cuenta del mudo físico y generar comunidades de aprendizaje, y en la educación superior aproximación a los desarrollos contemporáneos de las ciencias y participación en grupos y centros de investigación- resulta fundamental que en el debate acerca de los usos sociales de las ciencias, de los alcances y límites de la investigación y del tipo de necesidades que se generan y que se satisfacen por medio de los productos del trabajo científico, participe una comunidad filosófica que conozca y comprenda a la ciencia en su desarrollo, evolución y compromiso político y social.

Si para ser competitivo en el mercado un país o una región requiere de científicos comprometidos con el desarrollo de una ciencia ética y contextualmente consistente, para garantizar dicha consistencia se requiere de la combinación de perspectivas para definir tanto los destinos profesionales como los horizontes educativos de este grupo de profesionales.

UNESCO (2008b) define la filosofía como una "escuela de libertad," cuyo propósito es propiciar el cuestionamiento y liberar las mentes jóvenes por medio del ejercicio del libre pensamiento y de la reflexión sistemática de la realidad. Los filósofos latinoamericanos tenemos en la educación una gran oportunidad para impactar esas mentes jóvenes a las que se espera que liberemos. Se espera que cumplamos con una tarea que hemos heredado. Ahora hace falta que la asumamos como propia, como inescapable, como definitoria.

2 Véase: Eslava, 2014. 
Dussel, E. (2006). Filosofía de la cultura y la liberación. México D.F.: UACM.

Eslava, E. (2014). Conceptos, pertinencia y acceso: una mirada filosófica a la educación en ciencias naturales. Revista Colombiana de Filosofía de la Ciencia, 14(28), pp. 67-82.

Fensham, P. (2008). Science Education Policy-making. Eleven Emerging Issues. UNESCO.

Fuentes, C. (2012). Carlos Fuentes, del otro lado del espejo. El Comercio. Consultado el 12.12.14. Disponible en línea: http://www.elcomercio.com/tendencias/cultura/carlos-fuentes-del-lado-del.html

Nussbaum, M. (1993). Non-relative Virtues: An Aristotelian Approach. M. Nussbaum \& A. Sen (Eds.), The Quality of Life (pp. 242-269). New York: Oxford University Press.

Nussbaum, M. (1997). Cultivating Humanity. A Classical Defence of Reform in Liberal Education. Cambridge, MA: Harvard University Press.

OECD. (2011). Compendium of OECD Well-being Indicators.

OEI. (2008). Metas educativas 2021. La educación que queremos para la generación de los bicentenarios.

Sen, A. (1980). Equality of What. S.M. McMurrin (ed.), The Tanner Lectures on Human Values (pp. 196-220). Salt Lake City: University of Utah Press.

UNESCO. (2007a). Educación de Calidad para Todos. Un asunto de derechos humanos. Politicas educativas dentro del marco de la II Reunión Intergubernamental del Proyecto Regional en Educación para América Latina y el Caribe (EFA/PRELAC).

UNESCO. (2007b). The State of Education in Latin America and the Caribbean: Guaranteeing Quality Education for All. A Regional Report, Reviewing and Assessing the Progress toward Education for All within the Framework of the Regional Education Project (EFA/PRELAC).

UNESCO. (2008a). Panorama regional. América Latina y el Caribe.

UNESCO. (2008b). Philosophy, a School of Freedom. Teaching Philosophy and Learning to Philosophize: Status and Prospects. 
UNESCO. (2011). Regional Report on Education for All in Latin America and the Caribbean.

UNESCO. (2014a). Gasto Público en la educación en América Latina. ¿̨Puede servir a los propósitos de la Declaración de París sobre los Recursos Educativos Abiertos?

UNESCO. (2014b). Temas criticos para formular politicas docentes en América Latina y el Caribe: el debate actual.

UNESCO. (2014c). Philosophy Manual. A South-South Perspective.

Walker, M. \& Unterhalter, E. (Eds.). (2007) Amartya Sen's Capability Approach and Social Justice in Education. New York: Palgrave/McMillan.

Zea, L. (1953). América como conciencia. México D.F.: UNAM. 